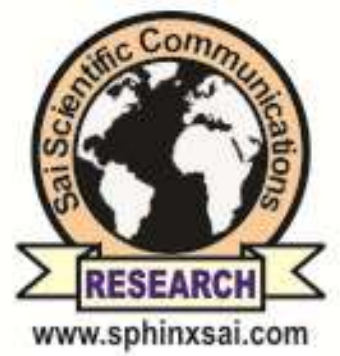

International Journal of PharmTech Research CODEN (USA): IJPRIF, ISSN: 0974-4304, ISSN(Online): 2455-9563 Vol.10, No.02, pp 114-119,

2017

\title{
Determination of Enantiomeric Methylphenidate in Human Plasma by High Performance Liquid Chromatography
}

\author{
José M.M. Batista ${ }^{1,2 \star}$, Lívia A.F. Guimarães ${ }^{1}$, Janete E.S. Soares ${ }^{3}$, \\ Marta M.F. Fonteles ${ }^{1,4}$, Teresa M.J.P. Carvalho ${ }^{3}$ \\ ${ }^{1}$ Department of Pharmacy, Faculty of Pharmacy, Dentistry and Nursing, Federal \\ University of Ceará, Ceará, Brazil \\ ${ }^{2}$ Pharmacy Graduation Course, Universitary Center of Quixadá - Unicatholic, \\ Ceará, Brazil \\ ${ }^{3}$ Department of Clinical Toxicological Analysis, School of Pharmacy, Dentistry and \\ Nursing, Federal University of Ceará,Ceará, Brazil \\ ${ }^{4}$ Postgraduate Program in Development and Technological Innovation in \\ Medicines, Federal University of Ceará,Ceará, Brazil
}

\begin{abstract}
Methylphenidate (MPD) is a chiral drug with stimulant action, used for the treatment of attention deficit hyperactivity disorder (ADHD) and narcolepsy. It has been used improperly and illegally, as it is assumed to improve cognitive performance. It was established a method of determining methylphenidate isomers in human plasma by separating racemic methylphenidates from the mixture using high performance liquid chromatography (HPLC) with a diode/UV detector. The separation was performed on a Chirobiotic V2 column, a mobile phase of methanol/ammonium acetate $(92: 8, V / V ; 20 \mathrm{mM} p H 4.1)$ and flow rate of 1 $\mathrm{mL} \min ^{-1}$; retention times were 7.0 and $8.1 \mathrm{~min}$ for $l$-MPD and $d$-MPD, respectively, detected at $215 \mathrm{~nm}$. The HPLC method was validated via linearity, precision, accuracy and recovery. The method was suitable for separation and determination of enantiomers of methylphenidate in human plasma and is considered precise and accurate.
\end{abstract}

Keywords: methylphenidate, enantiomers, HPLC-DAD/UV.

Introduction

Methylphenidate (MPD) is a piperidine derivative, methyl phenyl(piperidin-2-yl)acetate, related to amphetamines. It is a sympathomimetic that is heterogeneously distributed in the brain, with higher concentrations in the striatum, cerebral cortex, and cerebellum. The pharmacological mechanism of action is dopamine reuptake inhibition, without increasing neurotransmitter release $\mathrm{e}^{\mathbf{1 - 2}}$. MPD contains two chiral centers, being a mixture of four stereoisomers, the $(d, l)$-threo and $(d, l)$-erythro forms. The racemate $(d, l)$-threo-MPD (Ritalin, Concerta ${ }^{\circledR}$ ) is used to treat attention deficit hyperactivity disorder (ADHD) and narcolepsy ${ }^{3}$, being marketed as a racemic mixture (50:50) of $d$-threo-( $R, R)$ - and $l$-threo-MPD $(S, S)$-MPD ${ }^{4-5}$ (Fig. 1). 
Studies on the chirality of methylphenidate in humans report that pre-systemic metabolism and clearance of $(d, l)$-threo-MPD is an enantioselective process resulting in higher plasma concentrations, longer $t_{1 / 2}$ and higher pharmacological activity of $d$-threo-MPD compared to $l$-threo-MPD. This is due to the preference of carboxylesterase for $l$-threo-MPD. The half-life of racemic methylphenidate is 2-4 hours with a 3-5-hour duration of action. The predominant metabolic path generates ritalinic acid, an inactive product formed by esterification mediated by the enzyme carboxylesterase- $1^{4-5}$.It is well known that the enantiomers can cause different pharmacological and pharmacokinetic effects; therefore, clinical and pharmacological studies of enantiomeric forms are of the utmost importance ${ }^{6}$.

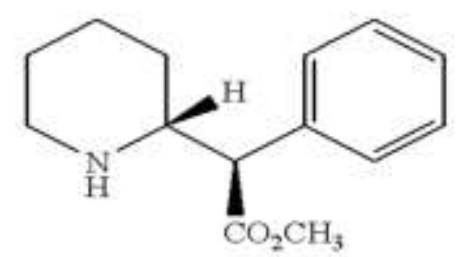

d-threo-Methylphenidate

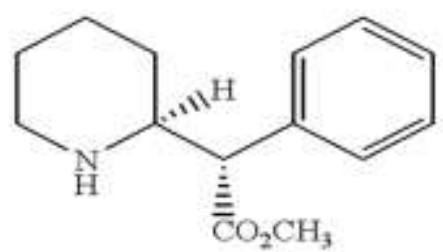

1-threo-Methylphenidate

Fig. 1. Chemical structures of Methylphenidate isomers.

The development of analytical methods that can assist in the identification, quantification, control and monitoring of chiral drugs in biological fluids is increasingly necessary. Several methods have been used to analyze plasma methylphenidate; notable among them is high-performance liquid chromatography with a diode array detector ${ }^{7-8}$.

Advanced chromatographic techniques enable the detection of substances of interest, as well as the determination of plasma concentrations, in populations ranging from patients to athletes, professionals and students. As such, chromatography can be a technological alternative for both clinical and non-therapeutic assessment and monitoring9. This method may be an important pathway in the context of drug addiction and abuse $^{\mathbf{1 0 - 1 5}}$, toxicity investigations and forensic applications ${ }^{16-17}$.

This study is aimed to develop and validate a bioanalytical method for enantiomeric determination of methylphenidate in human plasma using HPLC-DAD/UV.

\section{Experimental}

\section{Materials and reagents}

The following reagents were used: methylphenidate (Cerriliant, USA), acetonitrile, cyclohexane (Vetec, Brazil).Methanol, acetic acid, ammonium acetate, sodium bicarbonate and sodium carbonate (J. T. Baker, USA). All reagents were of analytical or HPLC grade. The water was purified with a quality $18 \Omega$ - System ElgaPurelab Option (Brazil). Reference standards were of $>98 \%$ purity. A stock solution of methylphenidate racemate (Cerriliant, USA) was prepared in methanol, at a concentration of $1000 \mu \mathrm{g} \mathrm{mL}^{-1}$.

\section{Sample preparation}

Blank plasma samples from healthy volunteers (not treated with methylphenidate) were obtained from the Blood Center of the Federal University of Ceará, Brazil (HEMOCE-UFC). Blank plasma samples and quality controls were treated as follows: $500-\mu \mathrm{L}$ aliquots of human plasma were alkalinized with $50 \mu \mathrm{L}$ of sodium carbonate buffer $\left(1\right.$ mole $\left.\mathrm{L}^{-1}\right)$, extracted with $2 \mathrm{~mL}$ of cyclohexane in a shaker $\left(\right.$ WARMNEST $^{\circledR}$, 
Curitiba-PR, Brazil)for 2 minutes and centrifuged in a Rotofix 32 (HETTICH ZENTRIFUGEN ${ }^{\circledR}$, Germany) at $6,000 \mathrm{rpm}$ for 10 minutes. The organic phase was separated, transferred to conic tube, and evaporated dry under vacuum to give a residue, which was reconstituted in $100 \mu \mathrm{L}$ of methanol and transferred to a vial, from which $25-\mu \mathrm{L}$ aliquot was injected into the chromatograph.

\section{Chromatographic conditions}

The chromatographic analysis employed a system comprised of an AcellaHPLC (Thermo Scientific, USA) with an auto-injector, coupled to a PDA/UV visible detector with variable wavelength. ChromQuest 5.0 (Thermo Scientific) was used for data collection and processing of chromatograms.

The analytical chiral chromatography column used for the separation of methylphenidate enantiomers were resolved on a CHIROBIOTIC V2 column $(150 \times 4.6 \mathrm{~mm})$ packed with 5 - $\mu \mathrm{m}$ particles (Sigma-Aldrich, USA) using a C18 guard column (Phenomenex, USA) for isocratic conditions. The mobile phase consisted of methanol/ammonium acetate $(92: 08, \quad V / V, \quad 20 \mathrm{mM}, \quad \mathrm{pH}$ 4.1). The flow rate was 1 $\mathrm{mL} \mathrm{min}^{-1}$ and the injection volume was $25 \mu \mathrm{L}$. UV detection was made at $215 \mathrm{~nm}$.

All mobile phases mixtures were filtered through a vacuum-pump filtration system (GAST, USA), PTFE membrane (0.45-0.47 $\mu \mathrm{m})$ (Pall, Brazil) and homogenized in an ultrasound bath (Q335D model, QUIMIS, Brazil).

\section{Validation}

The method developed for the analysis of enantiomers of methylphenidate in human plasma has been validated in accordance with the recommendations of National Health Surveillance Agency (Brazil) ${ }^{\mathbf{1 8}}$ for the validation of bioanalytical methods. This included testing for selectivity, specificity, linearity, precision and accuracy.

Calibration curves were obtained using $25 \mu \mathrm{L}$ of each standard solution $(1000,800,400,200,100$, and $50 \mu \mathrm{g} \mathrm{mL}^{-1}$ ) rac-methylphenidate in methanol and $500 \mathrm{uL}$ of the free drug plasma concentration. We obtained solutions for each isomer the following concentrations: 1.25, 2.5, 5.0, 10.0, 20.0 and $25.0 \mu$ gof each isomer $\mathrm{mL}$ plasma, which underwent the same preparation procedure as the samples.

The quality control samples (CQ) were used to validate and monitor the method performance. The high concentration quality control (HQC) was $80 \%$ of the highest concentration calibration curve $(20 \mu \mathrm{g}$ of each isomer $\mathrm{mL}^{-1}$ plasma), low concentration quality control (QCL) was two times the quantification limit $(2.5 \mu \mathrm{g}$ of each isomer $\mathrm{mL}^{-1}$ plasma) and the medium quality control (CQM) was $10 \mu \mathrm{g}$ of each isomer $\mathrm{mL}^{-1}$ plasma.

Specificity of the method was assessed by direct comparison of the areas of the peaks of methylphenidate injected directly in mobile phase and spiked post extraction into extracts originating from six different sources of human plasma. The linearity of the method was determined by the analysis of plasma samples spiked with the increasing analyte concentrations (in triplicate) in relation to the ones used for the construction of the calibration curve.

The methylphenidate recovery was evaluated in triplicate $\left(2.5,10\right.$ and $20 \mu \mathrm{g} \mathrm{mL} L^{-1}$ plasma of each isomer), by comparing the areas of the peaks obtained after plasma extraction with the areas of the peaks obtained when the standards were spiked into blank human plasma extracts ${ }^{19}$.

The quantification limit $(L O Q)$ was obtained by the analysis in quintuplicate of plasma samples spiked with methylphenidate racemate solution, at concentrations as low as $1.25 \mu \mathrm{g} \mathrm{mL} \mathrm{L}^{-1}$ human plasma of each isomer. The $L O Q$ was defined as the lowest plasma concentration of each stereoisomer analyzed with defined precision and accuracy (error of $20 \%$ or lower).

The precision and accuracy were determined by analyzing plasma samples spiked with three concentrations of methylphenidate isomers $(2.5,10$ and $20 \mu \mathrm{g} \mathrm{mL}$ plasma for each isomer).Precision was established using the Relative Standart Deviation (RSD\%). Intra-assay and inter-assay precision and accuracy were evaluated in a single day or on three consecutive days, respectively, using the quality controls. High quality control (HQC) comprised $20 \mu \mathrm{g}$ of each isomer per ml plasma; medium quality control (MQC) 
comprised $10 \mu \mathrm{g}$ of each isomer per ml plasma; low quality control (LQC) comprised $2.5 \mu \mathrm{g}$ of each isomer per $\mathrm{ml}$ plasma. Precision was evaluated based on the variation Relative Standart Deviation (RDS\%), and accuracy was based on the Relative Standard Error (RSE).

Aliquots of each spiked plasma sample were stored at $-20{ }^{\circ} \mathrm{C}$ and analyzed in replicate experiments $(n=5)$ using a calibration curve for intra-assay evaluation. Aliquots of each spiked plasma sample were analyzed in replicate experiments on three consecutive assays for inter-assay evaluation $(n=15)$, using a calibration curve for each assay.

\section{Results and discussion}

\section{Method development}

The method developed to analyze methylphenidate isomers in plasma samples used liquid-liquid extraction with cyclohexane in approx. 1 hour ${ }^{20-21}$. Elution was achieved at reasonable retention times, 7.0 and $8.1 \mathrm{~min}$ for $L$-MPD and $D$-MPD, respectively ${ }^{22}$ (peaks 1 and 2, Fig. 2). The reverse-phase chromatographic method was adequate in the conditions reported.

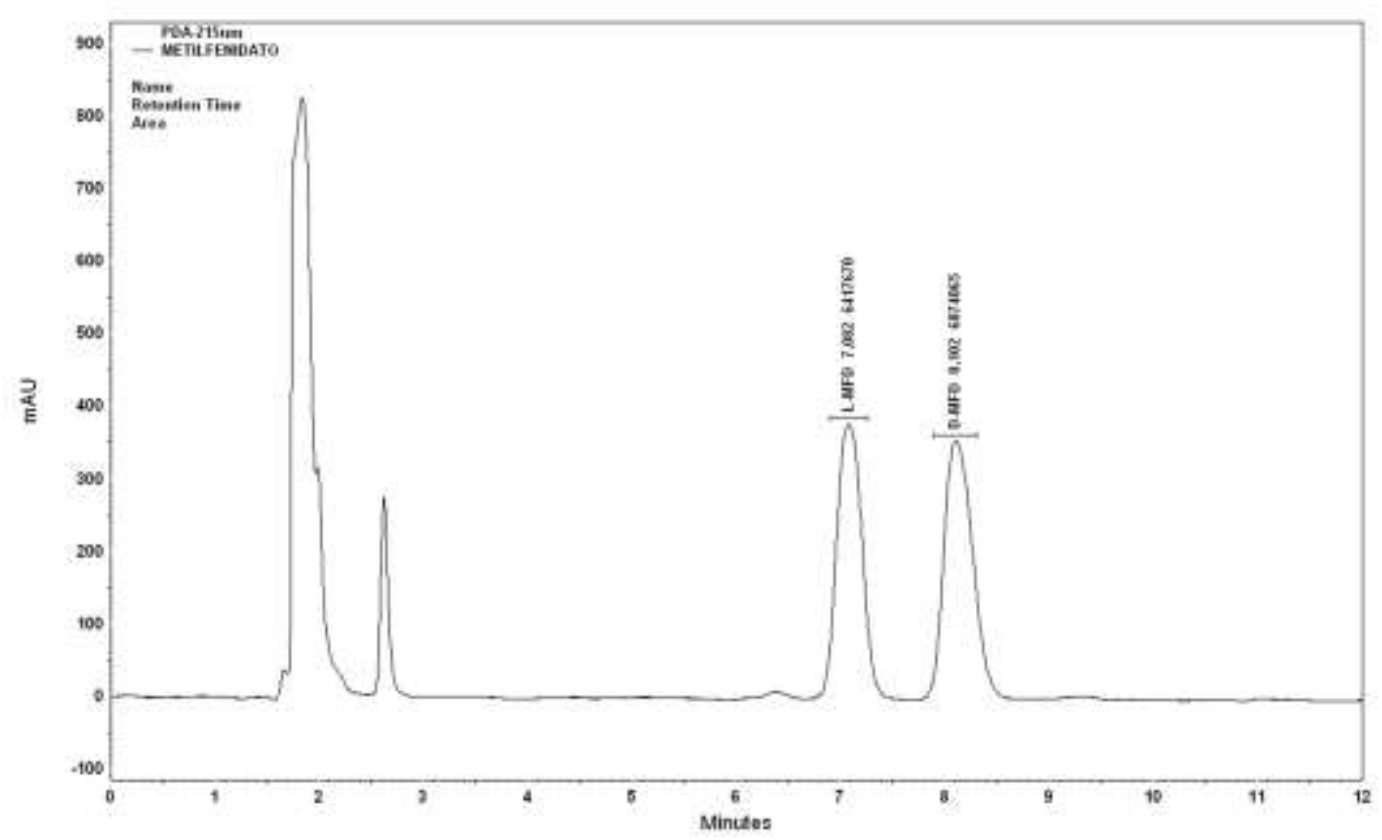

Fig. 2. HPLC enantioseparation of Methylphenidate racemate.

\section{Validation}

Linearity - $L O Q$. - The wavelength most suitable for compound quantification was $215 \mathrm{~nm}$. Calibration curves were constructed by correlating the area and concentration using six concentrations ranging from 1.25 to 25.0 $\mu \mathrm{gmL}^{-1}$ plasma for $L$-and $D$-MPD.Simultaneous assays were run and quantification was found to be linear on five separate days. The linearity was satisfactory for both enantiomers; coefficients of correlation were $\mathrm{R} \geq 0.997$ and $R^{\prime} \geq 0.987$. The recovery method was $80 \%$ for L-MPD and $75 \%$ for D-MPD.

Precision and accuracy - Precision was established where RDS was 9.1 and 11.9 for L-MPD and D-MPD, respectively. Accuracy was verified by the following RSE values 8.2 and -1.7 for L-MPD and D-MPD, respectively. The results are not only within established acceptable limits of $\pm 20 \%$ (Table 1), but also the values found are below $\pm 15 \%$ (Table 2). 
Table 1: Precision and accuracy results for the limit of quantification (LOD)

\begin{tabular}{|l|c|c|}
\hline \multicolumn{3}{|c|}{} \\
\hline \multicolumn{3}{|c|}{ Limit of Quantification (LOQ) $\mathbf{1 . 2 5} \boldsymbol{\mu g}$ of each isomer/ml plasma } \\
\hline Precision (RSD\%) & L-MPD & D-MPD \\
\hline Accuracy (RSE) & 9.1 & 11.9 \\
\hline
\end{tabular}

$R S D \%$ (Relative Standart Deviation $)=($ standard deviation $/$ mean $) * 100 ;$ RSE $=[($ Conc experimental - Conc real) / Conc. Real] $x 100$

Table 2: Enantiomeric method of plasma methylphenidate validation parameters

\begin{tabular}{|l|c|c|c|c|}
\hline \multicolumn{5}{|c|}{ Intra-assay (N=5) } \\
\hline & L-MPD & D-MPD & L-MPD & D-MPD \\
\hline LQC & \multicolumn{3}{|c|}{ Precision (RSD\%) } & \multicolumn{2}{c|}{ Accuracy (RSE) } \\
\hline MQC & 6.6 & 6.4 & -7.92 & 3.12 \\
\hline HQC & 4.2 & 5.7 & -2.1 & 0.1 \\
\hline \multicolumn{5}{|c|}{ Inter-assay (N=15) } \\
\hline LQC & 4.7 & 12.9 & -11.7 & -4.6 \\
\hline MQC & 11 & 14.5 & -8.5 & -1.7 \\
\hline HQC & 12.9 & 7.6 & -6.4 & 5.5 \\
\hline
\end{tabular}

LQC (Low Quality Control - $2.5 \mathrm{mg} / \mathrm{ml}$ ); MQC (Medium Quality Control - $10 \mu \mathrm{g} / \mathrm{ml}$ ); HQC (High Quality Control - $20 \mu \mathrm{g} / \mathrm{ml})$; RSE (Relative Standard Error $)=[($ Conc experimental - Conc real $) /$ Conc. Real $] \times 100$; $\mathrm{RSD} \%($ Relative Standart Deviation $)=($ standard deviation $/$ mean $) * 100$

This method for separation of methylphenidate enantiomeric will be of utmost importance in pharmacokinetic studies. These would enable to further understand the role (toxicity or therapeutic effect) of each of the isomers. Additionally, this method with a quantification limit of $1.25 \mu \mathrm{g} \mathrm{mL}^{-1}$ for each isomer in plasma could be an interesting alternative for the identification and monitoring in case of poisoning by methylphenidate as well as in possible forensic investigations ${ }^{23-24}$, doping or drugs abuse ${ }^{\mathbf{1 1}}$.

\section{Conclusions}

We have established a high performance liquid chromatography/UV method for the separation and quantification of methylphenidate enantiomers in human plasma. The method is fast, accurate and precise. This HPLC-UV method can be adapted to liquid chromatography-mass spectrometry (LC-MS), allowing for higher efficiency in the quantitative determination of enantiomers of methylphenidate for the purpose of therapeutic drug monitoring.

\section{References}

1. N.D. Volkow, G.J. Wang, J.S. Fowler and Y.S. Ding, Biol. Psychiatry, 57, 1410 (2005); doi:10.1016/j.biopsych.2004.11.006.

2. T.E. Wilens, J. Clin. Psychopharmacol., 28(Suppl. 2), S46 (2008); doi:10.1097/JCP.0b013e318173312f.

3. F.M.S. Coelho, R.M. Elias, M. Pradella-Hallinan, L.R.A. Bittencourt and S. Tufik, J. Clin. Psychiatry, 34, 133 (2007).

4. J.A. Dopheide and S.R. Pliszka, Pharmacotherapy, 29, 656 (2009); doi:10.1592/phco.29.6.656.

5. J.S. Markowitz and K.S. Patrick, J. Clin. Psychopharmacol., 28, S54 (2008); doi:10.1097/JCP.0b013e3181733560.

6. L.A. Nguyen, H. He andC. Pham-Huy, Int. J. Biomed. Sci., 2, 85 (2006). 
7. M. Wada, K. Abe, R. Ikeda, R. Kikura-Hanajiri, N. Kuroda and K. Nakashima, Anal. Bioanal. Chem., 400, 387 (2011); doi:10.1007/s00216-011-4713-0.

8. S. Pokkula, S. Thota, V.R. Kumar and V.K. Nagabandi, Int. J. PharmTech. Res., 6, 462 (2014).

9. B. Stegmann, A. Dorfelt and E. Haen, Ther. Drug Monit., 38, 98 (2016); doi:10.1097/FTD.0000000000000245.

10. S. Djezzar, M.A. Courné and N. Richard, Eur. Psychiatry, 29, 1 (2014); doi:10.1016/S09249338(14)78097-6.

11. C. Beyer, C. Staunton and K. Moodley, BMC Med. Ethics, 15, 20 (2014); doi:10.1186/1472-6939-15-20.

12. C. Itaborahy and F.O. Ortega, Cienc Saúde Coletiva, 18, 803 (2013).

13. G. Finger, E.R. Silva and A. Falavigna, Rev. Assoc. Med. Bras., 59, 285 (2013); doi:10.1016/j.ramb.2012.10.007.

14. B. Maher, Nature, 452, 674 (2008); doi:10.1038/452674a.

15. R.R. Silveira, B. Lejderman, P.E.M.S. Ferreira and G.M.P. Rocha, Trends Psychiatry Psychother., 36, 101 (2014); doi:10.1590/2237-6089-2013-0065.

16. K. Deventer, K. Roels, F.T. Delbeke and P. Van Eenoo, Anal. Bioanal. Chem., 401, 421 (2011); doi:10.1007/s00216-011-4863-0.

17. T. Trenque, E. Herlem, M. Abou Taam and M. Drame, Springerplus, 3, 286 (2014); doi:10.1186/2193$\underline{1801-3-286 .}$.

18. Agência Nacional de Vigilância Sanitária - ANVISA, 2012, Resolução no 27: Validação de métodos bioanalíticos, 17 de maio de 2012, Brasília (2012).

19. N.M. Cassiano, J.C. Barreiro, L.R.R. Martins, R.V. Oliveira and Q.B. Cass, Quim. Nova, 32, 1021 (2009); doi:10.1590/S0100-40422009000400033.

20. M.H. Teicher, A. Polcari, M. Foley, E. Valente, C.E. McGreenery, W.-W. Chang, G. McKay and K.K. Midha, J. Child Adolesc. Psychopharmacol., 16, 416 (2006); doi:10.1089/cap.2006.16.416.

21. D. Tuerck, S. Appel-Dingemanse, M. Maboudian, F. Pommier, Y. Wang and G. Sedek, J. Clin. Pharmacol., 47, 64 (2007); doi:10.1177/0091270006293757.

22. H. Zhu, J. Chromatogr. A, 879, 783 (2011).

23. T. Jansen, L.C.G. Hoegberg, J.W. Gregersen, I. Filipovski and S.S. Johansen, J. Clin. Toxicol., 3, 153 (2013).

24. K. Klampfl, A. Quattlander, R. Burger, B. Pfuhlmann, A. Warnke and M. Gerlach, Atten. Defic. Hyperact. Disord., 2, 221 (2010); doi:10.1007/s12402-010-0032-0. 